Article

\title{
Facile Synthesis of Yolk/Core-Shell Structured TS-1@Mesosilica Composites for Enhanced Hydroxylation of Phenol
}

\author{
Houbing Zou ${ }^{1,2, \dagger}$, Qingli Sun ${ }^{2, \dagger}$, Dongyu Fan ${ }^{1, *}$, Weiwei Fu ${ }^{2,3}$, Lijia Liu ${ }^{2}$ and Runwei Wang ${ }^{2, *}$ \\ 1 School of Science, Beijing University of Posts and Telecommunications, No. 10, Xitucheng Road, \\ Haidian District, Beijing 100876, China; E-Mail: zouhoubing@126.com \\ 2 State Key Laboratory of Inorganic Synthesis and Preparative Chemistry, College of Chemistry, \\ Jilin University, 2699 Qianjin Street, Changchun 130012, China; E-Mails: sqldudu@126.com (Q.S.); \\ fuweiwei1984@126.com (W.F.); liulijia_jiajia@163.com (L.L.) \\ 3 College of Chemistry, Experimental Center of Shenyang Normal University, \\ Shenyang 110034, China
}

$\dagger$ These authors contributed equally to this work.

* Authors to whom correspondence should be addressed; E-Mails: vandy@bupt.edu.cn (D.F.); rwwang@jlu.edu.cn (R.W.); Tel.: +86-10-6228-1949 (D.F.); Fax: +86-10-6228-5075 (D.F.); Tel./Fax: +86-431-8516-8115 (R.W.).

Academic Editor: Andreas Martin

Received: 26 October 2015 / Accepted: 23 November 2015 / Published: 14 December 2015

\begin{abstract}
In the current work, we developed a facile synthesis of yolk/core-shell structured TS-1@mesosilica composites and studied their catalytic performances in the hydroxylation of phenol with $\mathrm{H}_{2} \mathrm{O}_{2}$ as the oxidant. The core-shell TS-1@mesosilica composites were prepared via a uniform coating process, while the yolk-shell TS-1@mesosilica composite was prepared using a resorcinol-formaldehyde resin (RF) middle-layer as the sacrificial template. The obtained materials were characterized by X-ray diffraction (XRD), $\mathrm{N}_{2}$ sorption, Fourier transform infrared spectoscopy (FT-IR) UV-Visible spectroscopy, scanning electron microscopy (SEM) and transmission electron microscopy (TEM). The characterization results showed that these samples possessed highly uniform yolk/core-shell structures, high surface area $\left(560-700 \mathrm{~m}^{2} \mathrm{~g}^{-1}\right)$ and hierarchical pore structures from oriented mesochannels to zeolite micropores. Importantly, owing to their unique structural properties, these composites exhibited enhanced activity, and also selectivity in the phenol hydroxylation reaction.
\end{abstract}


Keywords: hierarchically zeolites composites; yolk/core-shell structured TS-1 zeolite; hydroxylation of phenol

\section{Introduction}

Hydroxylation of phenol with hydrogen peroxide $\left(\mathrm{H}_{2} \mathrm{O}_{2}\right)$ as oxidant has been widely applied for the production of two useful compounds, viz. catechol and hydroquinone [1,2]. Catechol is used as agrichemical, and in the synthesis of aromatic compounds, whereas hydroquinone is used as an antioxidant in elastics pigment. Various homogeneous and heterogeneous catalysts have been developed to achieve hydroxylation, including Fenton's reagent [3], Fe-containing molecular sieves [4-7], $\mathrm{Cu}$-containing materials [8], and Ti-containing porous materials [9-14]. Among these functional materials, TS-1 zeolite is one of the most efficient solid catalysts with high activity and selectivity coupled with environmentally benign catalytic performance $[13,14]$. However, the small pores in this zeolite make the diffusion of substrate and products through the channel difficult, thereby leading to limited catalytic performance. Therefore, many studies were directed toward improving the accessibility of the active sites in TS- 1 catalysts. To this end, introduction of a second mesopore into TS-1 zeolite crystals to form hierarchical structured TS-1 is an efficient strategy [15-21]. While significant achievements have been realized in this approach, the methods employed usually involved either cost-intensive mesostructured templates [15,19-21] or complex synthetic procedures [17,18]. In this regard, synthesis of TS-1 zeolite-based composites is an alternative approach.

Recently, hierarchical core-shell structured composites with a microporous zeolite crystal core and a mesoporous silica/carbon shell have received much attention [22-27] and have been widely applied in the some important catalytic reactions, such as epoxidation of alkene [23], cyclohexanone ammoximation [24], methanol to propylene (MTP) conversion [22] and catalytic cracking of large molecules [25]. Owing to the unique structural properties and the bimodal pore structure with open junction and retainable diffusion efficiency, these zeolite-based core-shell composite materials exhibit superior catalytic activity and selectivity. Moreover, multifunctional core-shell composites with various catalytic sites have been also designed to catalyze one pot cascade reactions. For-example, $\mathrm{Rh}(\mathrm{OH})_{3}$ species were supported on the core-shell structured TS-1@KCC-1 composite for one-pot synthesis of benzamide from benzaldehyde, ammonia and hydrogen peroxide [28]. However, TS-1 zeolite-based yolk/core-shell composites have been rarely reported for application in hydroxylation of phenol. In the current work, we devoted our efforts to study the catalytic properties of TS-1 zeolite-based yolk/core-shell composites in the hydroxylation of phenol and determine the relationship between the mircostructure of the material and its catalytic performance.

To study the catalyzed hydroxylation of phenol, specifically we prepared yolk/core-shell structured TS-1@mesosilica composites and used $\mathrm{H}_{2} \mathrm{O}_{2}$ as the oxidant. The core-shell structured TS-1@mesosilica composite was prepared via a uniform coating process, whereas the yolk-shell structured TS-1@mesosilica composite was prepared, for the first time, using a resorcinol-formaldehyde resin (RF) middle-layer as the sacrificial template. The obtained materials were characterized by X-ray diffraction (XRD), $\mathrm{N}_{2}$ 
sorption, FT-IR, UV-Visible spectroscopy, scanning electron microscopy (SEM) and transmission electron microscopy (TEM).

\section{Results and Discussion}

\subsection{Synthesis and Characterization of CS-TS-1@mSiO 2}

The micro-sized TS-1 single crystal was prepared by hydrothermal method. The SEM image (Figure 1a) and TEM image (Figure 2a,b) indicate that the TS-1 sample consist of uniform monodisperse nanocrystals with a particle size of 290-350 nm. Core-shell structured TS-1@mesosilica composites (designated as CS-TS-1@ $\mathrm{mSiO}_{2-x}, x$ is the thickness of $\mathrm{mSiO}_{2}$ layer) were obtained through a uniform coating process using water-ethanol (2:1), ammonia aqueous, CTAB (hexadecyltrimethylammonium bromide) and TEOS (tetraethylorthosilicate) as solvent, basic catalyst, surfactant and precursor, respectively $[29,30]$. The SEM micrograph (Figure 1b) shows that the particles are oval in shape with a large diameter (350-400 nm), suggesting that an $\mathrm{mSiO}_{2}$ layer of approximately $30 \mathrm{~nm}$ was formed on the surface of the TS-1 crystals. From the TEM image in Figure 2d, it can be clearly seen that every TS- 1 crystal is encapsulated with a $30 \mathrm{~nm} \mathrm{mSiO} 2$ layer. Furthermore, the HRTEM images in Figure 2e,f reveal that the mesopores are nearly perpendicular to the microporous frameworks (directed by red arrows) and there are close connections between the mesoporous silica shells and the zeolite cores, facilitating efficient transportation of reactants into active microporous framework through the mesochannels. It is worth mentioning that the current coating process did not involve any surface modifiers or additions that were often necessary for obtaining uniform core-shell structured zeolite@ $\mathrm{mSiO}_{2}$ composites with oriented mesochannels in $\mathrm{mSiO}_{2}$ shell $[22,23]$, which can be attributed to the flexibility in current synthesis system [29,30]. In addition, the thickness of mesoporous silica shells could be tailored by adjusting the amount of TEOS precursor. For example, when the amount of TEOS was increased to $0.20 \mathrm{~mL}$, we obtained CS-TS-1@ $\mathrm{mSiO}_{2}$ with a uniform $\mathrm{mSiO}_{2}$ shell of $50 \mathrm{~nm}$ (Figure $2 \mathrm{~g}-\mathrm{i}$ ). This control on thickness of the $\mathrm{mSiO}_{2}$ shell is significant in regulating the catalytic properties of materials.
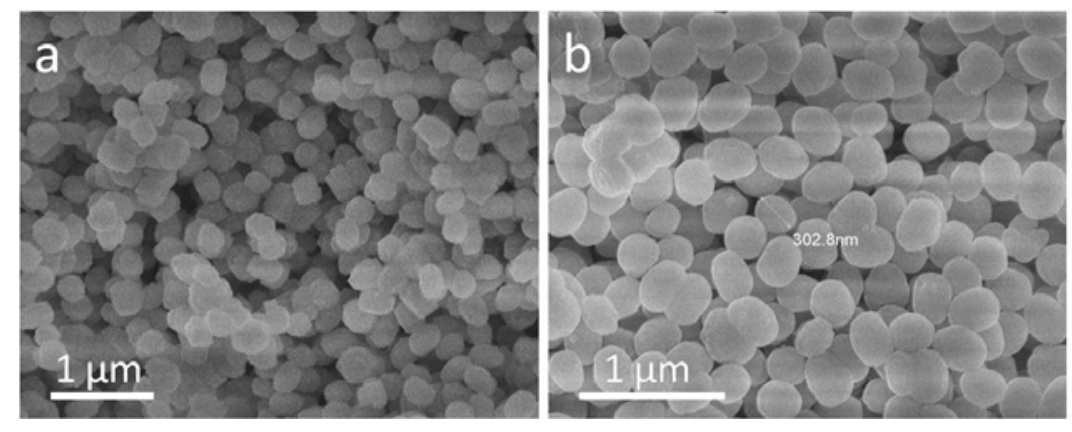

Figure 1. (a) SEM images of pristine TS-1; (c) CS-TS-1@ $\mathrm{mSiO}_{2}-50$.

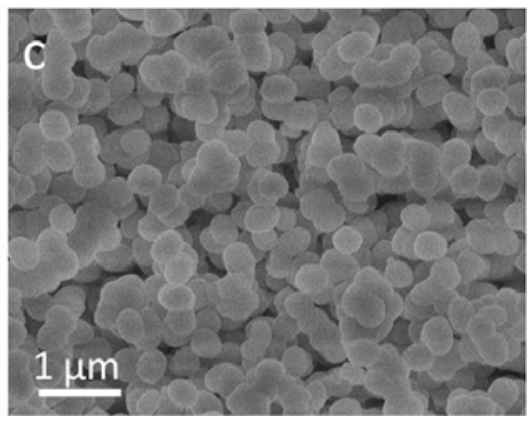

(b) CS-TS-1@mSiO $2-30$ and 

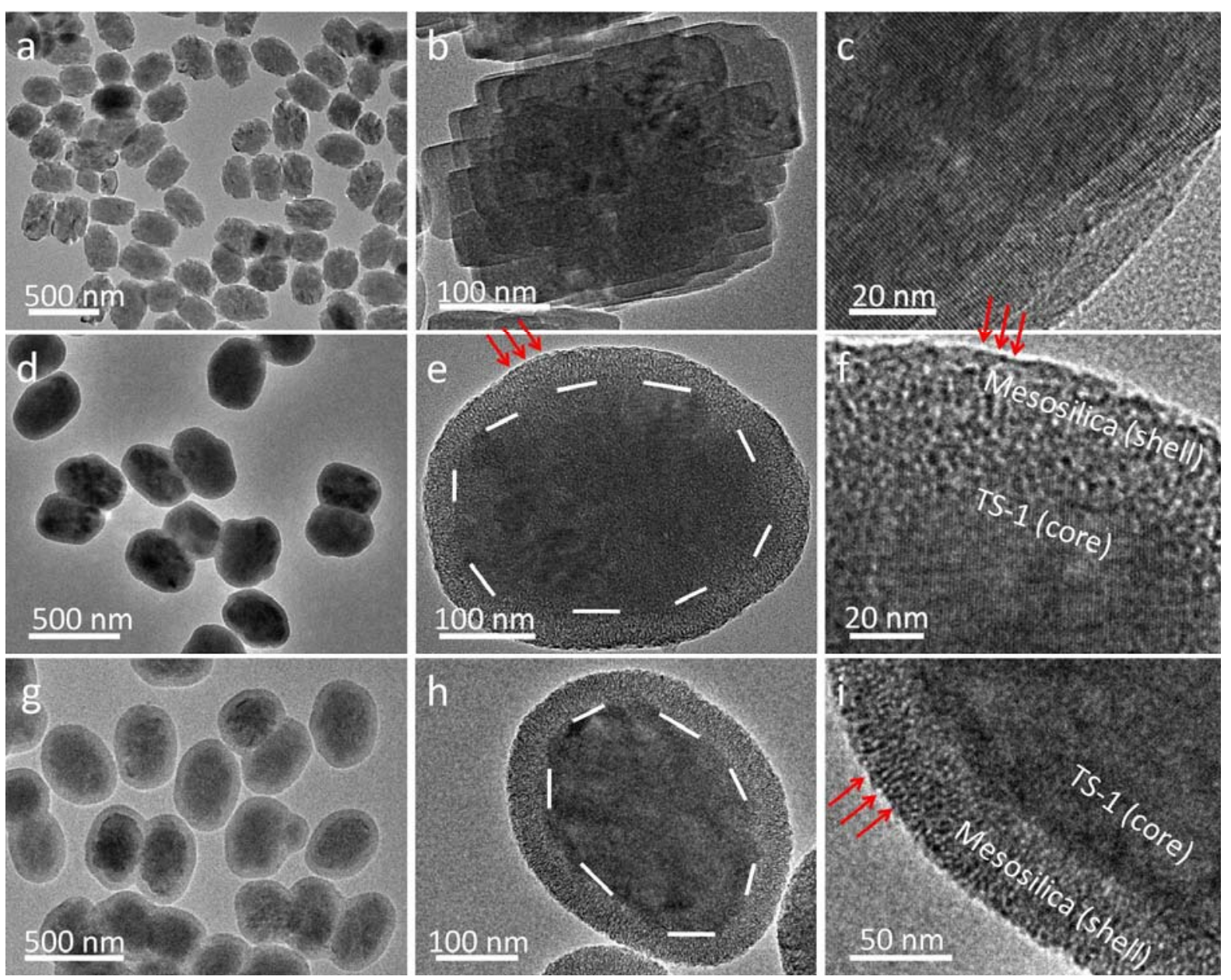

Figure 2. Low- moderate- and high-magnification TEM images of (a-c) pristine TS-1; (d-f) CS-TS-1@ $\mathrm{mSiO}_{2}-30$ and (g-i) CS-TS-1@ $\mathrm{mSiO}_{2}-50$.

The nanoporous structure of CS-TS-1@ $\mathrm{mSiO}_{2}$ composites was further characterized by XRD and nitrogen sorption analysis. Apparently, the small-angle XRD pattern of CS-TS-1@mSiO2-30 (Figure 3A) shows a broad diffraction peak at a $2 \theta$ value of approximately $2.4^{\circ}$ whereas pristine TS- 1 shows no diffraction peaks. This suggests an unordered mesostructure in $\mathrm{mSiO}_{2}$ shell. Moreover, a broad diffraction peak with higher intensity and a weak diffraction peak are simultaneously present at $2 \theta$ values of $2.4^{\circ}$ and $4.8^{\circ}$ in the small-angle XRD pattern of CS-TS-1@ $\mathrm{mSiO}_{2}-50$ (Figure 3A), revealing that the mesochannels in $\mathrm{mSiO}_{2}$ shell are partly ordered. These results indicate that the order degree of the mesochannels increased with increase in the thickness of them $\mathrm{SiO}_{2}$ shell, which is in accordance with the TEM results and was likely caused due to the increase in the domain-size of the ordered mesostructure [25]. The wide-angle XRD patterns of both the composites (Figure 3B) exhibit the same characteristic diffraction peaks as those of the pristine TS-1, assigned to the typical MFI structure. The lower diffraction intensity is probably due to the shielding effects of the mesosilica shell.

In the $\mathrm{N}_{2}$ sorption analysis, the pristine TS-1 shows a type-I isotherm according to the IUPAC (International Union of Pure and Applied Chemistry) classification with a sharp uptake in the $P / P_{0}$ range $0-0.01$, a characteristic behavior of completely microporous materials (Figure 3C). On the other hand, the CS-TS-1@ $\mathrm{mSiO}_{2}$ composites with different shell thickness (30 nm or $50 \mathrm{~nm}$ ) exhibit a similar uptake at low $P / P_{0}$ (type-I curves); however, an additional uptake following typical type-IV curves with a 
capillary condensation step appears at moderate $P / P_{0}(0.2-0.6)$. A distinct hysteresis loop of $\mathrm{H} 2$ type is also clearly observed for both the composites. These phenomena reveal the bimodal-pore properties of the CS-TS-1@ $\mathrm{mSiO}_{2}$ composites from micropores to mesopores as well as the accessible microporous ZSM-5 cores covered with a mesoporous silica shell. Moreover, in comparison with CS-TS-1@mSiO2-30, a higher uptake in the moderate $P / P_{0}$ of $0.2-0.6$ and a more obvious $\mathrm{H} 2$ type hysteresis loop in the $P / P_{0}$ of 0.4-0.6 are apparent for the CS-TS-1@ $\mathrm{mSiO}_{2}-50$, further suggesting the intrinsic bimodal-pore properties and the tunable mesopore shells. The pore size distribution (PSD) of pristine TS-1 reflects undetectable mesopores and a distinct micropore with a diameter of $0.55 \mathrm{~nm}$. By contrast, both the CS-TS-1@ $\mathrm{mSiO}_{2}$ show two distinct PSD models corresponding to micropores with a similar size to pristine TS-1 (around $0.55 \mathrm{~nm}$ ) and mesopores with a Barrett-Joyner-Halenda (BJH) diameter centered at $2.6 \mathrm{~nm}$, respectively (Figure 3D), which indicates the formation of uniform hierarchical pores. Both the CS-TS-1@ $\mathrm{mSiO}_{2}$ composites exhibit a high Brunauer-Emmett-Teller (BET) surface area of $\geq 550 \mathrm{~m}^{2} \mathrm{~g}^{-1}$ of which the microporous surface area was $\geq 50 \mathrm{~m}^{2} \mathrm{~g}^{-1}$ (Table 1), and a high total pore volume of $\geq 0.45 \mathrm{~m}^{3} \mathrm{~g}^{-1}$. The lower micropore surface area was possibly due to presence of a low portion of TS-1 in the composites. All the above results indicate that a highly uniform core-shell structured TS-1@ $\mathrm{mSiO}_{2}$ composite with tunable shell thickness was successfully synthesized via a simple coating process.
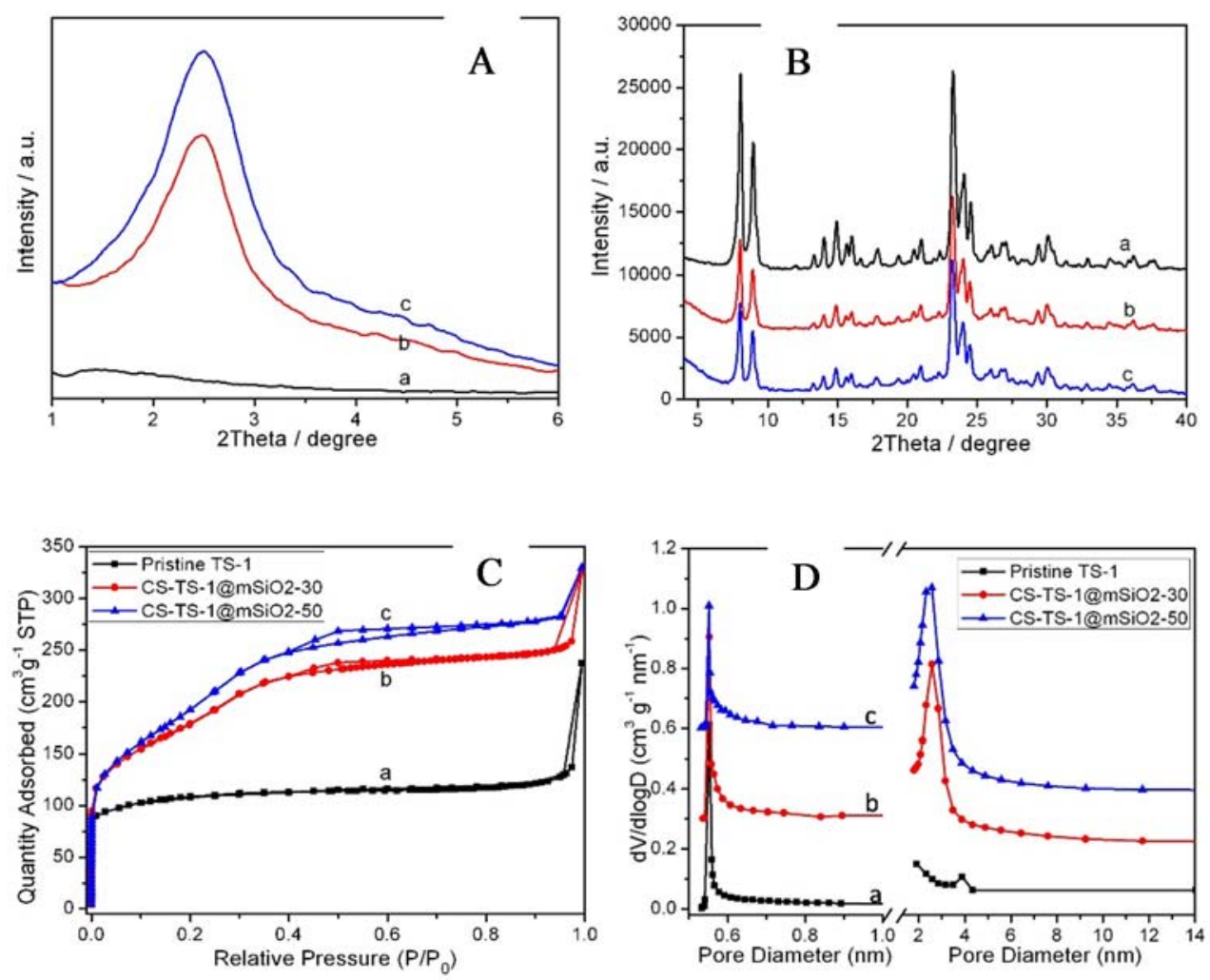

Figure 3. (A) Small-angle XRD patterns of (a) pristine TS-1, (b) CS-TS-1@ $\mathrm{mSiO}_{2}-30$ and (c) CS-TS-1@ $\mathrm{mSiO}_{2}-50$; (B) wide-angle XRD patterns of (a) pristine TS-1, (b) CS-TS-1@mSiO2-30 and (c) CS-TS-1@ $\mathrm{mSiO}_{2}-50$; (C) $\mathrm{N}_{2}$ sorption isotherms; (D) $\mathrm{HK}$ and $\mathrm{BJH}$ pore size distribution curves. 
Table 1. .Textural properties of pristine TS-1, core-shell structured TS-1@mesosilica composite (CS-TS-1@mSiO2) and yolk-shell structured TS-1@mesosilica composite (YS-TS-1@mSiO2) ${ }^{\mathrm{a}}$.

\begin{tabular}{|c|c|c|c|c|c|c|c|}
\hline Sample & $\begin{array}{c}S_{\mathrm{BET}} \\
{\left[\mathbf{m}^{2} \mathbf{g}^{-1}\right]} \\
\end{array}$ & $\begin{array}{c}S_{\text {micro }} \\
{\left[\mathrm{m}^{2} \mathbf{g}^{-1}\right]} \\
\end{array}$ & $\begin{array}{c}S_{\text {ext }} \\
{\left[\mathbf{m}^{2} \mathbf{g}^{-1}\right]}\end{array}$ & $\begin{array}{c}V_{\text {micro }} \\
{\left[\mathrm{m}^{3} \mathrm{~g}^{-1}\right]} \\
\end{array}$ & $\begin{array}{c}V_{\text {ext }} \\
{\left[\mathbf{m}^{3} \mathbf{g}^{-1}\right]}\end{array}$ & $\begin{array}{l}D_{\text {micro }} \\
{[\mathrm{nm}]}\end{array}$ & $\begin{array}{l}D_{\text {meso }} \\
{[\mathrm{nm}]}\end{array}$ \\
\hline pristine TS-1 & 368.5 & 249.3 & 119.2 & 0.12 & 0.26 & 0.55 & - \\
\hline CS-TS-1@mSiO $2-30$ & 564.6 & 71.8 & 492.8 & 0.031 & 0.412 & 0.55 & 2.6 \\
\hline CS-TS-1@mSiO $2-50$ & 635.9 & 53.2 & 582.7 & 0.027 & 0.454 & 0.55 & 2.6 \\
\hline YS-TS-1@mSiO ${ }_{2}$ & 695.2 & 57.2 & 638.0 & 0.028 & 0.484 & 0.55 & 2.8 \\
\hline
\end{tabular}

The nature and coordination of Ti species in silica network was characterized by FT-IR and UV-Vis spectroscopy. The FT-IR spectrum of pristine TS-1 shows three different bands at $550 \mathrm{~cm}^{-1}, 804 \mathrm{~cm}^{-1}$ and $960 \mathrm{~cm}^{-1}$ (Figure 4A). The band at 550 and $804 \mathrm{~cm}^{-1}$ are assigned to $\delta(\mathrm{Si}-\mathrm{O}-\mathrm{Si})$ and $v(\mathrm{Si}-\mathrm{O}-\mathrm{Si})$, respectively. The peak at $960 \mathrm{~cm}^{-1}$ is often referred to as Ti-O-Si band and can be considered as a good indication of titanium substitution into the zeolite framework. In the UV-vis spectrum (Figure 4B), the maximum absorption peak appears around $210 \mathrm{~nm}$, which further confirms the existence of the $\mathrm{Ti}$ framework. Furthermore, the presence of a weak absorption at $330 \mathrm{~nm}$ suggests the presence of a low amount of anatase phase. Importantly, both the CS-TS-1@ $\mathrm{mSiO}_{2}$ exhibit identical FT-IR and UV-Vis spectra as those of the pristine TS-1, revealing that the Ti active species was retained in the TS- 1 core during the coating process. Additionally, the absence of the bands at 250-280 nm in these UV-Vis spectra reveals that amorphous Ti species is neither present in the TS-1 core nor in the $\mathrm{mSiO}_{2}$ shell. These results indicate that the process of coating $\mathrm{mSiO}_{2}$ did not have any negative effect on the catalytic behavior of TS-1.
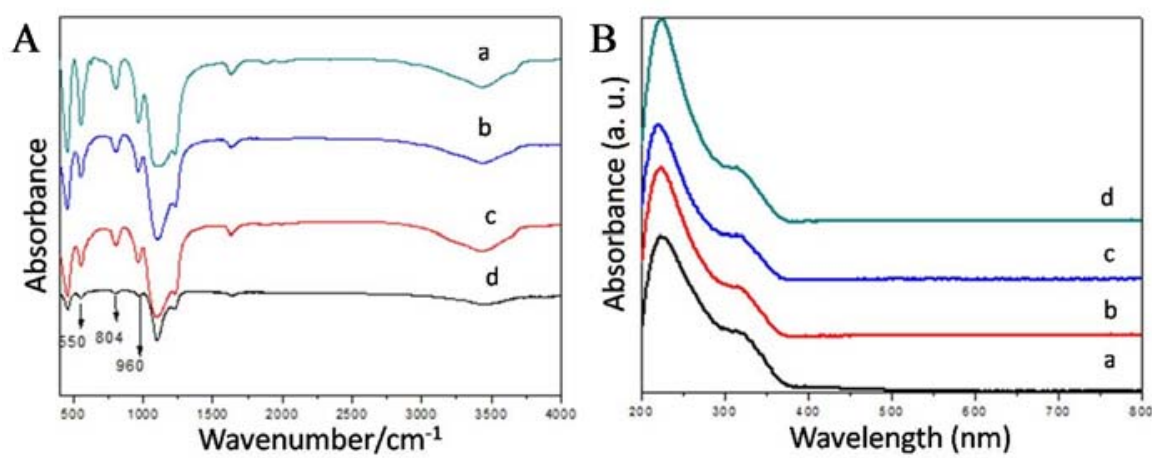

Figure 4. (A) FT-IR spectra and (B) UV-Vis spectra of (a) pristine TS-1, (b) CS-TS-1@mSiO2-30, (c) CS-TS-1@mSiO $2-50$ and (d) YS-TS-1@ $\mathrm{mSiO}_{2}$.

\subsection{Synthesis and Characterization of YS-TS-1@mSiO}

The yolk-shell structured TS-1@mesosilica composite (YS-TS-1@mSiO ${ }_{2}$ ) was prepared using a resorcinol-formaldehyde resin (RF) middle-layer as the sacrificial template. Following a published report [31], the TS-1 crystals were first coated with the RF layer via simple cooperative template-directed 
coating method to form core-shell structured TS-1@RF. A successful coating was confirmed from the large decrease in the nitrogen uptake at low $P / P_{0}(0-0.01)$ (Figure 5e), and the microporous surface area from $249.3 \mathrm{~m}^{2} \mathrm{~g}^{-1}$ to $36 \mathrm{~m}^{2} \mathrm{~g}^{-1}$ of the synthesized TS-1@RF. A similar coating for CS-TS-1@mSiO2-30 was also done to obtain sandwich type TS-1@RF@mSiO2. This was followed by the removal of the middle-layer RF, surfactant CTAB and structure direct agent TPA ${ }^{+}$by calcination at $550{ }^{\circ} \mathrm{C}$ in air for $6 \mathrm{~h}$, which yielded YS-TS-1@ $\mathrm{mSiO}_{2}$ with an open hierarchical pore structure from the inherent micropore in TS-1 core and the mesopore in $\mathrm{mSiO}_{2}$ shell to nanocapsule. The sandwich type TS-1@RF@mSiO2 displays a similar oval sharp as that of CS-TS-1@ $\mathrm{mSiO}_{2}-30$ and a larger particle diameter (410-450 nm) (Figure 5a). By contrast, the obtained YS-TS-1@ $\mathrm{mSiO}_{2}$ exhibits a distinct yolk-shell structure after the simple calcination process in air (Figure 5b,c). One $\mathrm{mSiO}_{2}$ shell encapsulates one TS- 1 crystal and a void space of around $15 \mathrm{~nm}$ between the TS- 1 core and $\mathrm{mSiO}_{2}$ shell is clearly observed from the TEM images (Figure 5b,c). Moreover, the SEM image (Figure 5d) shows some broken particles indicated by white arrows, further confirming the yolk-shell structure. In addition, identical with the CS-TS-1@ $\mathrm{mSiO}_{2}$ composites, the YS-TS-1@ $\mathrm{mSiO}_{2}$ also exhibit a retained uptake at low $P / P_{0}$ (type-I curves) and typical type-IV curves with a capillary condensation step at moderate $P / P_{0}(0.2-0.6)$ as well as a distinct hysteresis loop of $\mathrm{H} 2$ type. On the other hand, the corresponding DFT PSD not only shows two sharp peaks centered at $0.55 \mathrm{~nm}$ and $2.8 \mathrm{~nm}$ that was ascribed to the micropores in TS-1 core and mesopores in $\mathrm{mSiO}_{2}$ shell, respectively, but also a broad small peak in the range of 10-20 nm, which resulted from the void space between the TS-1 core and the $\mathrm{mSiO}_{2}$ shell. This phenomenon is in good agreement with the TEM result. YS-TS-1@ $\mathrm{mSiO}_{2}$ shows a high BET surface area of $695 \mathrm{~m}^{2} \mathrm{~g}^{-1}$ (Table 1). All these results demonstrated that we were able to successfully prepare a

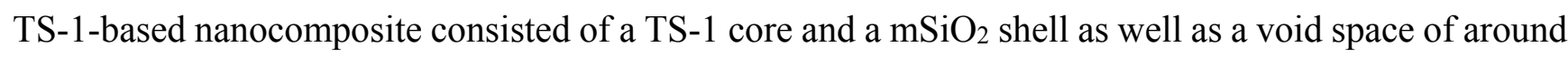
$15 \mathrm{~nm}$ between the core and the shell. The FT-IR and UV-Vis spectra (Figure 4A,B) also reveal that the nature and coordination of Ti species keeps identical with pristine TS-1.
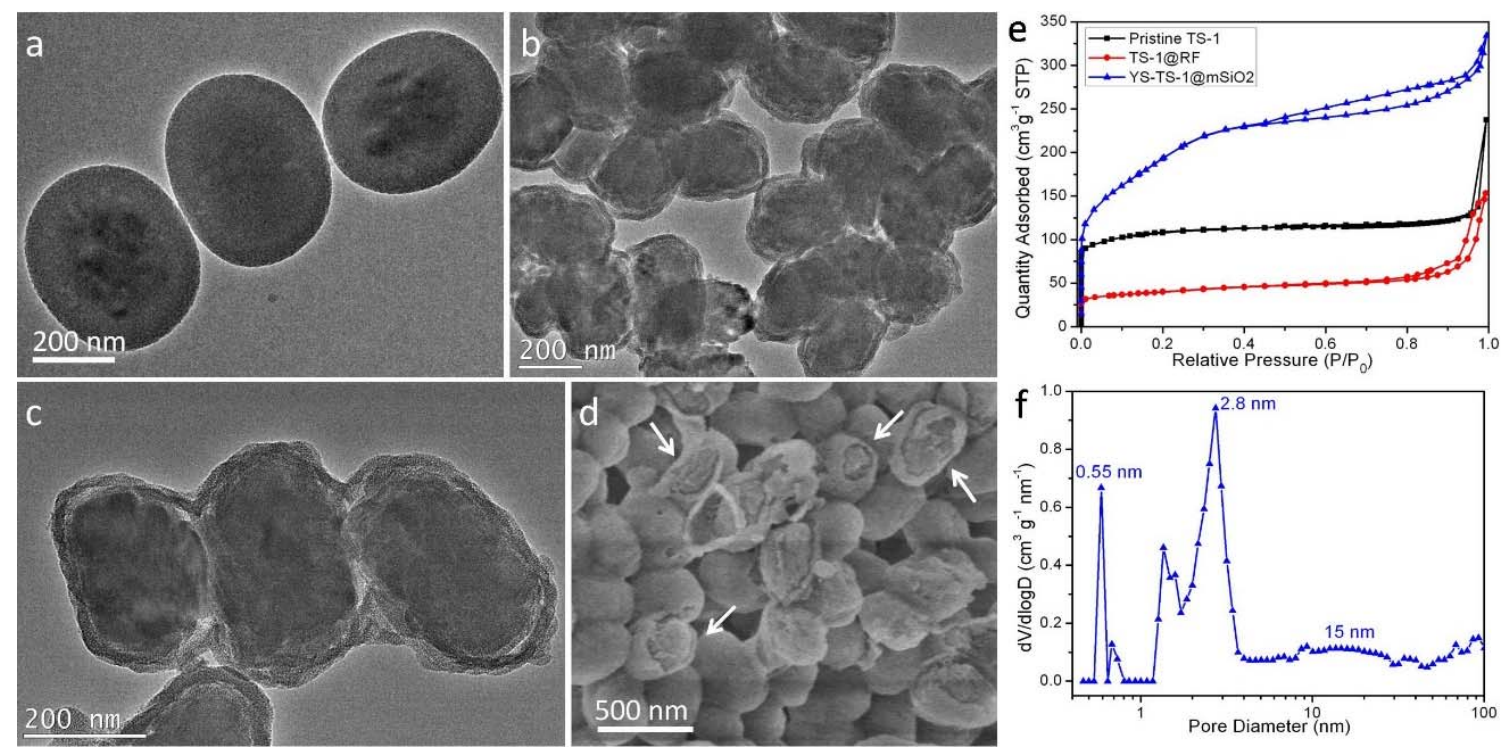

Figure 5. (a) TEM image of TS-1@RF@ $\mathrm{mSiO}_{2}$; (b-d) TEM images and SEM image of

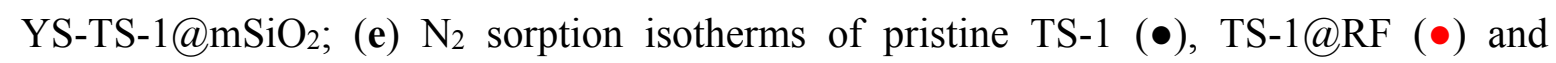
YS-TS-1@ $\mathrm{mSiO}_{2}(\boldsymbol{\Delta})$; (f) Relevant DFT pore size distribution curve of YS-TS-1@ $\mathrm{mSiO}_{2}$. 


\subsection{Catalytic Performance of CS/YS-TS-1@mSiO ${ }_{2}$ in Hydroxylation of Phenol}

Table 2 summarizes the catalytic results of various TS- 1 catalysts in the hydroxylation of phenol using $\mathrm{H}_{2} \mathrm{O}_{2}$ as the oxidant and water as the solvent. The pristine TS-1 material (Entry 3 ) showed similar activity and selectivity with previous reports $[12,13]$. Notably, our prepared composites CS-TS-1@ $\mathrm{mSiO}_{2}$ (Entry 6) and YS-TS-1@ $\mathrm{mSiO}_{2}$ (Entry 9) gave a higher phenol conversion of $25.2 \%$ and $27.6 \%$ than that of pristine TS-1 (23.7\%), respectively. The turnover frequency (TOF) of YS-TS-1@ $\mathrm{mSiO}_{2}(34.4)$ was even higher than some other hierarchical nanoporous TS-1 zeolite materials [18]. The enhanced activities should be attributed to the effect of external mesosilica layer that can effectively capture the large phenol molecules like a pump from the reaction solution due to the large specific surface area of mesopores. This "capture" effect was also reported for enhanced catalytic cracking reactions in previous publication [25]. Moreover, the void space between the $\mathrm{mSiO}_{2}$ shell and the TS-1 core reinforced this positive "capture" effect for further enhancing the catalytic activity of material. In addition, we were aware that the selectivity of side product benzoquinone was slightly increased from $3.9 \%$ to $5.5 \%$ using our composites as the catalysts, which indicated that the external mesosilica layer and the void space also adsorbed products catechol and hydroquinone when capturing substrate phenol from reaction solution, and thus led to further oxidation. Moreover, it is worth mentioning that more product hydroquinone was produced in the cases of using composites CS-TS-1@ $\mathrm{mSiO}_{2}$ and YS-TS-1@ $\mathrm{mSiO}_{2}$ as catalysts. These catalytic results clearly revealed that the external $\mathrm{mSiO}_{2}$ shell and the void space between the $\mathrm{mSiO}_{2}$ shell and the TS-1 core not only can capture substrate molecules like a pump for enhanced activity, but also adsorb product molecules, and thus posed diffusion resistance for negative selectivity.

Table 2. Catalytic activities of various TS- 1 catalysts in hydroxylation of phenol ${ }^{\text {a }}$.

\begin{tabular}{|c|c|c|c|c|c|c|c|c|}
\hline \multirow{2}{*}{ Entry } & \multirow{2}{*}{ Catalyst } & \multirow{2}{*}{$\begin{array}{c}\text { Reaction } \\
\text { Time }\end{array}$} & \multirow{2}{*}{ Conv. $/ \%$} & \multicolumn{3}{|c|}{ Product Sel. ${ }^{\mathrm{b}} / \%$} & \multirow{2}{*}{ HQ/CAT } & \multirow{2}{*}{$\operatorname{TOF}^{\mathrm{c}} / \mathbf{h}^{-1}$} \\
\hline & & & & CAT & HQ & BQ & & \\
\hline 1 & \multirow{3}{*}{ Pristine TS- 1} & $1 \mathrm{~h}$ & 6.2 & 10.3 & 1.5 & 88.2 & 0.15 & \multirow{3}{*}{29.6} \\
\hline 2 & & $3 \mathrm{~h}$ & 16.6 & 60.7 & 25.1 & 14.1 & 0.41 & \\
\hline 3 & & $5 \mathrm{~h}$ & 23.7 & 55.1 & 41.0 & 3.9 & 0.74 & \\
\hline 4 & \multirow{3}{*}{ CS-TS-1@mSiO } & $1 \mathrm{~h}$ & 18.7 & 55.8 & 24.5 & 19.7 & 0.44 & \multirow{3}{*}{31.5} \\
\hline 5 & & $3 \mathrm{~h}$ & 24.3 & 51.2 & 38.1 & 10.7 & 0.74 & \\
\hline 6 & & $5 \mathrm{~h}$ & 25.2 & 45.7 & 49.8 & 4.5 & 1.09 & \\
\hline 7 & \multirow{3}{*}{ YS-TS-1@mSiO } & $1 \mathrm{~h}$ & 20.7 & 49.7 & 24.1 & 26.2 & 0.48 & \multirow{3}{*}{34.4} \\
\hline 8 & & $3 \mathrm{~h}$ & 24.8 & 50.8 & 38.6 & 10.6 & 0.76 & \\
\hline 9 & & $5 \mathrm{~h}$ & 27.6 & 47.4 & 47.1 & 5.5 & 0.99 & \\
\hline
\end{tabular}

${ }^{a}$ Reaction conditions: water as solvent, $10 \mathrm{~mL}$; phenol, $1.0 \mathrm{~g}$ (10.63 mmol); phenol/ $\mathrm{H}_{2} \mathrm{O}_{2}$ molar ratio, 3.0; catalyst mass (containing TS-1), $40 \mathrm{mg}$ (0.017 mmol Ti); temperature, $80{ }^{\circ} \mathrm{C}$; reaction time, $5 \mathrm{~h}$. ${ }^{\mathrm{b}}$ CAT: catechol, HQ: hydroquinone, BQ: benzoquinone. ${ }^{\mathrm{c}}$ Turnover frequency (TOF): moles of phenol converted per mole of titanium per hour.

In order to obtain more details, we performed a kinetic study for the above three catalysts at different reaction times. Obviously, the reaction rate was YS-TS-1@ $\mathrm{mSiO}_{2}>\mathrm{CS}-\mathrm{TS}-1 @ \mathrm{mSiO}_{2}>$ pristine TS-1 (Entries 1-9). At the initial stage of reaction, because the $\mathrm{mSiO}_{2}$ shell and the void space could capture substrate molecules, the concentration of phenol around active Ti sites was YS-TS-1@ $\mathrm{mSiO}_{2}>$ CS-TS-1@ $\mathrm{mSiO}_{2}>$ pristine TS-1. Therefore, after reacting for $1 \mathrm{~h}$, the conversions of composite 
YS-TS-1@mSiO 2 and CS-TS-1@mSiO 2 were much higher than that of pristine TS-1 (20.7\% vs. 18.7\% vs. 6.2\%). Moreover, the decreasing rate of benzoquinone's selectivity of pristine TS-1 was faster than that of YS-TS-1@ $\mathrm{mSiO}_{2}$ and CS-TS-1@ $\mathrm{mSiO}_{2}$. This controlled experiment further confirmed aforementioned diffusion/adsorption effect. This will help us to design other multifunctional zeolites-based nanocomposites for various oxidation reactions.

\section{Experimental Section}

\subsection{Chemicals}

Tetraethylorthosilicate (TEOS, 98\%), aqueous ammonia $\left(\mathrm{NH}_{3} \cdot \mathrm{H}_{2} \mathrm{O}, \quad 28 \%\right.$ ) and cetyltrimethyl-ammonium bromide (CTAB, 99.0\%) were purchased from Sinopharm Chemical Reagent Co. Ltd., Shanghai, China. Tetrapropylammonium hydroxide $(20 \%-25 \%)$ was obtained from TCI, Shanghai, China. Hydrogen peroxide $\left(\mathrm{H}_{2} \mathrm{O}_{2}, 30 \%\right)$, phenol (AR) and tetrabutylorthotitanate (TBOT) were obtained from Beijing chemical works, Beijing, China. All chemicals were used as received without any further purification.

\subsection{Synthesis of Pristine TS-1 Zeolite}

In a typical synthesis, $8.0 \mathrm{~mL}$ of TPAOH, $5.0 \mathrm{~mL}$ of TEOS and $5.0 \mathrm{~mL}$ of water were mixed and stirred at room temperature for $24 \mathrm{~h}$ to form solution $\mathrm{A} ; 0.2 \mathrm{~mL}$ of TBOT was added into $10.0 \mathrm{~mL}$ of $30 \% \mathrm{H}_{2} \mathrm{O}_{2}$ aqueous to form solution $\mathrm{B}$. The solution $\mathrm{B}$ was added dropwise into solution A under stirring, and a clear sol was obtained after $24 \mathrm{~h}$. The obtained sol was taken in a Teflon container and placed in a stainless steel autoclave. The sol was hydrothermally treated at $100{ }^{\circ} \mathrm{C}$ for $24 \mathrm{~h}$. The TS- 1 zeolites were collected through centrifugation and then washed several times with water and ethanol.

\subsection{Synthesis of Core-Shell TS-1@mesosilica Composites}

The core-shell TS-1@mesosilica composite was prepared via a uniform coating process, according to our previous reports [29,30]. In a typical synthesis, $100 \mathrm{mg}$ of the as-prepared TS-1 zeolite was homogeneously dispersed in a mixture containing $22 \mathrm{~mL}$ of water and $11 \mathrm{~mL}$ of ethanol by ultrasonication for $30 \mathrm{~min}$. The resultant mixture was stirred at room temperature for $30 \mathrm{~min}$ followed by sequential addition of $0.12 \mathrm{~g}$ of CTAB, $0.25 \mathrm{~mL}$ of ammonium aqueous solution $(25 \%-28 \%)$, and $0.15 \mathrm{~mL}$ of TEOS. The as-obtained white mixture was further stirred at room temperature for $6 \mathrm{~h}$. The core-shell structured TS-1@mesosilica composites were collected by centrifugation and then washed with water and ethanol several times. Finally, the organic structure directing agent $\mathrm{TPA}^{+}$and the surfactant $\mathrm{CTAB}$ were removed by calcination at $550^{\circ} \mathrm{C}$ in air for $6 \mathrm{~h}$.

\subsection{Synthesis of Yolk-Shell TS-1@Mesosilica Composites}

The yolk-shell structured TS-1@mesosilica composite was prepared using an RF middle-layer as the sacrificial template. In a typical synthesis, $200 \mathrm{mg}$ of the as-prepared TS-1 zeolite was homogeneously dispersed in a mixture containing $17.5 \mathrm{~mL}$ of water and $11 \mathrm{~mL}$ of ethanol by ultrasonication for $30 \mathrm{~min}$. To the above solution were added $0.575 \mathrm{~g}$ of CTAB, $0.025 \mathrm{~mL}$ of ammonium aqueous solution 
$(25 \%-28 \%)$ and $0.12 \mathrm{~g}$ of resorcinol sequentially and the resultant mixture was stirred at $35{ }^{\circ} \mathrm{C}$ for $30 \mathrm{~min}$. Then, $0.3 \mathrm{~mL}$ of formalin solution was added to the dispersion under stirring maintaining $35^{\circ} \mathrm{C}$ for $6 \mathrm{~h}$. The mixture was cooled to room temperature after $6 \mathrm{~h}$, and then aged at room temperature overnight without stirring. The product (designated as TS-1@RF) was collected by centrifugation and then washed with water and ethanol several times. Next, a uniform mesosilica layer was coated on the surface of TS-1@RF similarly like TS-1@mesosilica to form TS-1@RF@mesosilica. Finally, the yolk-shell structured TS-1@mesosilica composite was obtained after removal of the middle-layer RF, surfactant $\mathrm{CTAB}$ and structure direct agent $\mathrm{TPA}^{+}$by calcination at $550{ }^{\circ} \mathrm{C}$ in air for $6 \mathrm{~h}$.

\subsection{Catalytic Tests}

In a typical catalytic reaction, desired amount of catalyst (containing $40 \mathrm{mg}$ of TS-1 zeolite), $1.0 \mathrm{~g}$ of phenol, $0.36 \mathrm{~mL}$ of $\mathrm{H}_{2} \mathrm{O}_{2}$ and $10 \mathrm{~mL}$ of $\mathrm{H}_{2} \mathrm{O}$ were mixed in a $50 \mathrm{~mL}$ round-bottomed flask equipped with a reflux condenser and a magnetic stirrer. After reaction at $80^{\circ} \mathrm{C}$ for $5 \mathrm{~h}$, the products were collected and analyzed by GC.

\subsection{Materials Characterization}

SEM images were taken with a JSM-6700F field-emission electron microscope (JEOL, Tokyo, Japan). TEM images were obtained from an Tecnai $\mathrm{G}^{2}$ F20s-twin D573 field emission transmission electron microscope (FEI, Hillsboro, FL, USA) at an accelerating voltage of 200kV. Powder XRD patterns were obtained by using a 2550 diffractometer (Rigaku, Tokyo, Japan) with $\mathrm{Cu}$ Ka radiation $(\lambda=1.5418 \AA) . \mathrm{N}_{2}$ adsorption-desorption isotherms were obtained at $-196{ }^{\circ} \mathrm{C}$ on a ASAP 2010 sorptometer (Micromeritics, Shanghai, China). Samples were degassed at $120{ }^{\circ} \mathrm{C}$ for a minimum of 12 $\mathrm{h}$ prior to analysis. BET surface areas were calculated from the linear part of the BET plot. Pore size distribution was estimated from the adsorption branch of the isotherm by the BJH method. The total pore volume was estimated from the adsorbed amount of nitrogen at $P / P_{0}=0.995$.

\section{Conclusions}

In summary, we have prepared two kinds of TS-1-based nanocomposites, namely, core-shell structured TS-1@mesosilica and yolk-shell structured TS-1@mesosilica. These nanocomposites showed highly uniform morphology and structure, high BET surface area, and hierarchical pore structures from oriented mesochannels to zeolite micropores. When used as catalysts in the hydroxylation of phenol with $\mathrm{H}_{2} \mathrm{O}_{2}$ as the oxidant, they exhibited enhanced activity and unique selectivity. We found that the external mesosilica shell and the void space between the mesosilica shell and the TS-1 core not only captured phenol molecules like a pump for enhanced activity but also posed diffusion resistance for negative selectivity. Moreover, these nanocomposites presented higher selectivity of the para-product. The current work is expected to offer some insights into designing of multifunctional zeolites-based nanocomposites for enhanced oxidation reactions. 


\section{Acknowledgments}

This work was supported by National Natural Science Foundation of China (21390394), the National Basic Research Program of China (2012CB821700, 2011CB808703), NSFC (21261130584, 91022030), “111" project (B07016), Award Project of KAUST (CRG-1-2012-LAI-009) and Ministry of Education, Science and Technology Development Center Project (20120061130012).

\section{Author Contributions}

Houbing Zou designed the experiments, analyzed the experimental data and wrote the manuscript. Qingli Sun prepared and characterized the samples. Lijia Liu tested the catalytic performances of samples. Weiwei Fu supported the experiments. Dongyu Fan and Runwei Wang revised the final version of paper.

\section{Conflicts of Interest}

The authors declare no conflict of interest.

\section{References}

1. Thangaraj, A.; Kumar, R.; Ratnasamy, P. Catalytic Properties of Crystalline Titanium Silicalites II. Hydroxylation of Phenol with Hydrogen Peroxide over TS-1 Zeolites. J. Catal. 1991, 131, 294-297.

2. Martens, J.A.; Buskens, P.; Jacobs, P.A.; van der Pol, A.; van Hooff, J.H.C.; Ferrini, C.; Kouwenhoven, H.W.; Kooyman, P.J.; van Bekkum, H. Hydroxylation of Phenol with Hydrogen Peroxide on EUROTS-1 Catalyst. Appl. Catal. A 1993, 99, 71-84.

3. Hamilton, G.; Friedman, J.; Campbell, P. The Hydroxylation of Anisole by Hydrogen Peroxide in the Presence of Catalytic Amounts of Ferric Ion and Catechol. Scope, Requirements, and Kinetic Studies. J. Am. Chem. Soc. 1966, 88, 5266-5268.

4. Villa, A.; Caro, C.; Correa, C. Cu- and Fe-ZSM-5 as Catalysts for Phenol Hydroxylation. J. Mol. Catal. A 2005, 228, 233-240.

5. Li, B.; Xu, J.; Liu, J.; Zuo, S.; Pan, Z.; Wu, Z. Preparation of Mesoporous Ferrisilicate with High Content of Framework Iron by pH-Modification Method and Its Catalytic Performance. J. Colloid Interface Sci. 2012, 366, 114-119.

6. Choi, J.-S.; Yoon, S.-S.; Jang, S.-H.; Ahn, W.-S. Phenol Hydroxylation Using Fe-MCM-41 Catalysts. Catal. Today 2006, 111, 280-287.

7. Liu, H.; Lu, G.; Guo, Y.; Guo, Y.; Wang, J. Study on the Synthesis and the Catalytic Properties of Fe-HMS Materials in the Hydroxylation of Phenol. Microporous Mesoporous Mater. 2008, 108, 56-64.

8. Fu, W.; Wang, R.; Wu, L.; Wang, H.; Wang, X.; Wang, A.; Zhang, Z.; Qiu S. Synthesis of $\mathrm{Cu}_{2}(\mathrm{OH}) \mathrm{PO}_{4}$ Crystals with Various Morphologies and Their Catalytic Activity in Hydroxylation of Phenol. Chem. Lett. 2013, 42, 772-774.

9. Clerici, M.G. Oxidation of Saturated Hydrocarbons with Hydrogen Peroxide Catalysed by Titanium Silicalite. Appl. Catal. 1991, 68, 249-261. 
10. Xiao, F.; Han, Y.; Yu, Y.; Meng, X.; Yang, M.; Wu, S. Hydrothermally Stable Ordered Mesoporous Titanosilicates with Highly Active Catalytic Sites. J. Am. Chem. Soc. 2002, 133, 888-889.

11. Lin, K.; Wang, L.; Meng, F.; Sun, Z.; Yang, Q.; Cui, Y.; Jiang, D.; Xiao, F.S. Formation of Better Catalytically Active Titanium Species in Ti-MCM-41 by Vapor-phase Silylation. J. Catal. 2005, 235, 423-427.

12. Tsai, S.-T.; Chao, P.-Y.; Tsai, T.-C.; Wang, I.; Liu, X.; Guo, X.-W. Effects of Pore Structure of Post-treated TS-1 on Phenol Hydroxylation. Catal. Today 2009, 148, 174-178.

13. Zhu, G.; Ni, L.; Qi, W.; Ding, S.; Li, X.; Wang, R. Synthesis and Morphology Research of Framework Ti-rich TS-1 Containing No Extraframework Ti Species in the Presence of $\mathrm{CO}_{2}$. Inorg. Chem. Commun. 2014, 40, 129-132.

14. Shi, C.; Chu, B.; Lin, M.; Long, J.; Wang, R. Cyclohexane Mild Oxidation Catalyzed by New Titanosilicate with Hollow Structure. Catal. Today 2011, 175, 398-403.

15. Cheneviere, Y.; Chieux, F.; Caps, V.; Tuel, A. Synthesis and Catalytic Properties of TS-1 with Mesoporous/microporous Hierarchical Structures Obtained in the Presence of Amphiphilic Organosilanes. J. Catal. 2010, 269, 161-168.

16. Moliner, M.; Corma, A. Advances in the synthesis of titanosilicates: From the medium pore TS-1 zeolite to highly-accessible ordered materials. Micropor. Mesopor. Mater. 2014, 189, 31-40.

17. Chen, L.; Li, X.; Tian, G.; Li, Y.; Rooke, J.; Zhu, G.; Qiu, S.; Yang, X.; Su, B. Highly Stable and Reusable Multimodal Zeolite TS-1 Based Catalysts with Hierarchically Interconnected Three-Level Micro-Meso-Macroporous Structure. Angew. Chem. Int. Ed. 2011, 50, 11156-11161.

18. Xin, H.; Zhao, J.; Xu, S.; Li, J.; Zhang, W.; Guo, X.; Hensen, E.; Li, C. Enhanced Catalytic Oxidation by Hierarchically Structured TS-1 Zeolite. J. Phys. Chem. C 2010, 114, 6553-6559.

19. Kang, Z.; Fang, G.; Ke, Q.; Hu, J.; Tang, T. Superior Catalytic Performance of Mesoporous Zeolite TS-1 for the Oxidation of Bulky Organic Sulfides. ChemCatChem 2013, 5, 2191-2194.

20. Na, K.; Jo, C.; Kim, J.; Ahn, W.; Ryoo, R. MFI Titanosilicate Nanosheets with Single-unit-cell Thickness as An Oxidation Catalyst Using Peroxides. ACS Catal. 2011, 1, 901-907.

21. Wang, R.; Liu, W.; Ding, S.; Zhang, Z.; Li, J.; Qiu S. Mesoporous MFI Zeolites with Self-stacked Morphology Templated by Cationic Polymer by Cationic Polymer. Chem. Commun. 2010, 46, 7418-7420.

22. Qian, X.; Du, J.; Li, B.; Si, M.; Yang, Y.; Hu, Y.; Niu, G.; Zhang, Y.; Xu, H.; Tu, B.; et al. Controllable Fabrication of Uniform Core-Shell Structured Zeolite@SBA-15 Composites. Chem. Sci. 2011, 2, 2006-2016.

23. Xu, L.; Ren, Y.; Wu, H.; Liu, Y.; Wang, Z.; Zhang, Y.; Xu, J.; Peng, H.; Wu, P. Core/shell-Structured TS-1@mesoporous Silica-supported Au Nanoparticles for Selective Epoxidation of Propylene with $\mathrm{H}_{2}$ and $\mathrm{O}_{2}$. J. Mater. Chem. 2011, 21, 10852-10858.

24. Xu, L.; Peng, H.; Zhang, K.; Wu, H.; Chen, L.; Liu, Y.; Wu, P. Core-Shell-Structured Titanosilicate As A Robust Catalyst for Cyclohexanone Ammoximation. ACS Catal. 2012, 3, 103-110.

25. Qian, X.; Li, B.; Hu, Y.; Niu, G.; Zhang, D.; Che, R.; Tang, Y.; Su, D.; Asiri, A.; Zhao, D. Exploring Meso-/Microporous Composite Molecular Sieves with Core-Shell Structures. Chem. Eur. J. 2012, 18, 931-939.

26. Lv, Y.; Qian, X.; Tu, B.; Zhao, D. Generalized Synthesis of Core-Shell Structured Nano-zeolite@ordered mesoporous Silica Composites. Catal. Today 2013, 204, 2-7. 
27. Li, C.; Lu, Y.; Wu, H.; Wu, P.; He, M. A Hierarchically Core/Shell-Structured Titanosilicate with Multiple Mesopore Systems for Highly Efficient Epoxidation of Alkenes. Chem. Commun. 2015, 51, 14905-14908.

28. Peng, H.; Xu, L.; Wu, H.; Zhang, K.; Wu, P. One-pot Synthesis of Benzamide Over A Robust Tandem Catalyst Based on Center Radially Fibrous Silica Encapsulated TS-1. Chem. Commun. 2013, 49, 2709-2711.

29. Zou, H.; Wang, R.; Li, X.; Wang, X.; Zeng, S.; Ding, S.; Li, L.; Zhang, Z.; Qiu, S. An Organosilane-directed Growth-induced Etching Strategy for Preparing Hollow/Yolk-shell Mesoporous Organosilica Nanospheres with Perpendicular Mesochannels and Amphiphilic Frameworks. J. Mater. Chem. A 2014, 2, 12403-12412.

30. Zou, H.; Wang, R.; Dai, J.; Wang, Y.; Wang, X.; Zhang, Z.; Qiu, S. Amphiphilic Hollow Porous Shell Encapsulated Au@Pd Bimetal Nanoparticles for Aerobic Oxidation of Alcohols in Water. Chem. Commun. 2015, 51, 14601-14604.

31. Guan, B.; Wang, X.; Xiao, Y.; Liu Y.; Huo, Q. A Versatile Cooperative Template-directed Coating Method to Construct Uniform Microporous Carbon Shells for Multifunctional Core-Shell Nanocomposites. Nanoscale 2013, 5, 2469-2475.

(C) 2015 by the authors; licensee MDPI, Basel, Switzerland. This article is an open access article distributed under the terms and conditions of the Creative Commons Attribution license (http://creativecommons.org/licenses/by/4.0/). 